\title{
Gallstones in Egyptian infants and children: risk factors, complications and outcome: a single center experience
}

\author{
A. Enayet ${ }^{*}$, R. A. Afifi, E. A. Mogahed, M. S. El-Raziky and M. A. K. Abdellatif
}

\begin{abstract}
Background: The widespread use of abdominal ultrasonography in children documented increased detection rate of gallstones. The aim of this study was to assess the possible risk factors of gallstones and to detect the complication rate and outcome of the disease in Egyptian infants and children from a hepatologist perspective. This prospective study included 35 cases, with ultrasound-detected gallstones, who presented to the Pediatric Hepatology Unit from July 2015 to October 2017. All patients were initially evaluated by full history, general and abdominal examination, laboratory assessment, and ultrasound findings to detect possible risk factors of gallstones. Management approach was individualized for each patient according to his/her condition, and the patients were followed up at 3 months interval.

Results: The patients' ages ranged from 3.5 months up to 17 years. The median age of gallstone detection was 2 years. Gallstones were symptomatic in $62.9 \%$ with abdominal pain in $51.4 \%$. Complications in the form of choledocholithiasis were detected in 7 patients (20\%). Laparoscopic cholecystectomy was done for 7 patients while conservative management was applied for 24 patients. Complete resolution occurred in $8.3 \%$ and partial resolution in $33.3 \%$. Chronic hemolytic anemia was the most commonly identified risk factor for gallstones. Patients needed surgical intervention had significant higher serum cholesterol and LDL levels ( $P=0.006$ and $P=0.002$ respectively).

Conclusion: Risk factors for gallstones could be identified in most of pediatric cases. Symptomatic presentation is common among the pediatric population. Conservative management is recommended for asymptomatic cases of gallstones in children. Laparoscopic cholecystectomy is a safe and recommended procedure for complicated and symptomatic cases of gallstones.
\end{abstract}

Keywords: Children, Complications, Gallstones, Infants, Outcome

\section{Background}

The prevalence of gallstones in the pediatric population has been reported to be uncommon with estimated overall prevalence of 0.13 to $1.9 \%$. However, the increased use of abdominal ultrasonography (US) in recent years has led to increased rate of detection of gallstones [1].

Many etiological factors have been associated with gallstones in children. Chronic hemolytic diseases are considered the most common cause. Other risk factors

*Correspondence: afaf_enayet@hotmail.com

Department of Pediatrics, Kasr Alainy Medical School, Faculty of Medicine, Cairo University, Cairo, Egypt

\section{Springer Open}

include liver cirrhosis, chronic cholestasis, total parenteral nutrition, and ileal diseases such as ileal resection, ileal Crohn's disease, cystic fibrosis, prolonged use of high-dose ceftriaxone, cancer therapy, family history, obesity, and congenital anomalies in the GB [2].

Little information is available about the management of this disorder in childhood as diagnostic approach, and therapeutic strategies are extremely heterogeneous. For symptomatic cases, cholecystectomy is curative, and laparoscopic cholecystectomy has been proven to be safe and efficient [3]. Asymptomatic patients show a more difficult management dilemma. Considering the low rate

(c) The Author(s). 2020 Open Access This article is licensed under a Creative Commons Attribution 4.0 International License, which permits use, sharing, adaptation, distribution and reproduction in any medium or format, as long as you give appropriate credit to the original author(s) and the source, provide a link to the Creative Commons licence, and indicate if changes were made. The images or other third party material in this article are included in the article's Creative Commons licence, unless indicated otherwise in a credit line to the material. If material is not included in the article's Creative Commons licence and your intended use is not permitted by statutory regulation or exceeds the permitted use, you will need to obtain permission directly from the copyright holder. To view a copy of this licence, visit http://creativecommons.org/licenses/by/4.0/. 
of complications detected by many studies, expectant management with periodical clinical and US follow-up is recommended. Spontaneous resolution of gallstones was observed in many cases [4]. Elective cholecystectomy is recommended for children with chronic hemolytic anemia at the time of gallstone diagnosis, before symptoms, or complications develop [5].

The aim of this study was to assess the possible risk factors of gallstones and to detect the complication rate and outcome of the disease in infants and children.

\section{Methods}

This prospective study was conducted at the Pediatric Hepatology Unit during the time period from July 2015 to October 2017. All cases below 18 years of age of both sexes with US detected gallstones were enrolled in this study. The study was approved by the institutional ethical committee of the Pediatrics Department. A written informed consent was taken from patients' guardians.

\section{Patient evaluation Initial diagnosis}

All patients were evaluated in terms of age, sex, presenting symptoms (typical biliary colic, nonspecific abdominal pain, nausea, vomiting or fatty food intolerance, and symptoms of complications as fever or jaundice), precipitating risk factor for gallstone, and other accompanying diseases.

\section{Laboratory assessment}

Complete blood count $(\mathrm{CBC})$ and liver function tests, hemolytic profile (reticulocytes, osmotic fragility, quantitative glucose 6 phosphate dehydrogenase (G6PD) measurement, hemoglobin electrophoresis, direct coombs test), lipid profile (serum cholesterol (LDL-HDL-VLDL) and serum triglycerides), and sweat chloride test if cystic fibrosis was suspected (multisystem presentations: pulmonary, gastrointestinal, and others as dehydration and metabolic alkalosis), and serum amylase and lipase if pancreatitis is suspected.

\section{Radiological assessment}

Abdominal US, endoscopic retrograde cholangiopancreatography (ERCP) in cases of choledocholithiasis, magnetic resonance cholangiopancreatography (MRCP) is done when choledocholithiasis is suspected, and ERCP cannot be done for technical consideration as young age (age less than 2 years) and in cases with US documented CBD dilatation.

\section{Management approach \\ Conservative management}

For asymptomatic patients with no complications, it involved periodic clinical and US follow up at 3-month intervals for at least 1 year.

\section{Medical dissolution of gallstones by Ursodeoxycholic Acid (UDCA)}

A trial of oral UDCA dissolution of stones was applied at a dose of $15 \mathrm{mg} / \mathrm{kg} /$ day for those patients with symptomatic uncomplicated gallstones with periodic clinical and ultrasound follow-up for symptoms' improvement and/or stone dissolution every 3 months. Symptomatic uncomplicated gallstones were defined as the presence of biliary colic in the form of significant abdominal pain in the absence of complications as acute or chronic cholecystitis, ascending cholangitis, common bile duct stones, or pancreatitis.

\section{ERCP}

ERCP is for patients with choledocholithiasis.

Laparoscopic cholecystectomy (LC) is for (1) patients with complicated gallstones as cases with choledocholithiasis, cholecystitis, or pancreatitis, (2) patients with symptomatic gallstones not improving on UDCA, and (3) patients with chronic hemolytic anemia.

\section{Patient follow-up}

Patients who were not indicated for surgery were followed every 3 months with clinical, laboratory and ultrasound assessments. Resolution of gallstones was based on ultrasonographic evidence of absence of gallbladder and bile duct stones.

\section{Statistical analysis}

Data was analyzed by the Statistical Package of Social Science Software program, version 23. Data was presented using mean, standard deviation (SD) for homogenous quantitative variables, median and interquartile range (IQR) for quantitative variables with nonhomogenous distribution, and frequency and percentage for qualitative ones. Comparison between groups was performed using Mann-Whitney test for quantitative variables and chi-square and Fisher's exact test for qualitative ones. $P$ values less than 0.05 were considered statistically significant.

\section{Results}

Thirty-five children with gallstones were included; $54.3 \%$ were males. Their age ranged from 3.5 months to 17 years. Out of these 35 patients, 18 patients $(51.4 \%)$ were below 2 years of age at the time of discovery of gallstones (infantile group), while 17 (48.6\%) had their discovery onset beyond 2 years. 
They were referred to hepatology clinic because of accidently detected gallstones in US done for other purposes in 25 cases (71.4\%), jaundice in 7 (20\%), and elevated liver enzymes in 3 (8.6\%). Patients with US detected gallstones were referred from everywhere including general outpatient clinics as well as hematology and neonatology units. US was repeated in hepatology clinic for confirmation and for detailed sonographic examination. Twenty-two (62.9\%) patients had symptomatic gallstones: abdominal pain as the most common presentation $(51.4 \%)$, jaundice $(28.6 \%)$, vomiting, nausea, and fatty food intolerance in $25.7 \%, 11.4 \%$, and $14.3 \%$ respectively. Eight patients (22.9\%) showed elevated total bilirubin; all with elevated direct fraction except one who had $\beta$-thalassemia major, in whom indirect bilirubin was elevated. The seven patients with direct hyperbilirubinemia included 6 patients with choledocholithiasis, and one patient had cholestasis with normal GGT.

Lipid profile showed abnormal results in 7 cases. Three patients (8.6\%) had hypercholesterolemia, and 4 patients had elevated triglycerides level.

Abdominal US done by 2 operators revealed single gallstones in 17 cases (48.6\%) and multiple gallstones in 18 cases (51.4\%). Size of stones ranged from 0.3 to 1.6 $\mathrm{cm}$ with median $0.6 \mathrm{~cm}$ with IQR $(0.4-0.8)$. Biliary sludge with gallstones was evident in 9 cases (25.7\%) while common bile duct (CBD) stones were detected in 6 cases (17.1\%); 5 (14.3\%) of them showed CBD and biliary radicles dilatation.
Possible etiology could be reached in 25 patients where hemolytic anemia was the most common identified diagnosis (Fig. 1). History of dehydration and neonatal intensive care admission were more prevalent among the infantile group but with no statistical significance. Duration of NICU admission was significantly longer among patients with multiple stones $(P=0.044)$ compared to those with single stone.

The management plan was individualized for each patient (Fig. 2). For cases with complicated gallstones in the form of choledocholithiasis, cholecystectomy was performed while conservative management was planned for the other 28 cases $(80 \%)$. The medical treatment was offered to non-hemolytic cases with patent cystic, and common bile duct with stone size was less than $2 \mathrm{~cm}$. LC was offered for patients with hemolytic anemia, before symptoms or complications developed, but unfortunately, it was not performed except in complicated cases.

LC was done for 7 patients (20\%): 6 of them had choledocholithiasis while one of the patients with choledocholithiasis dropped out of the study. A patient was indicated for LC because of attacks of agonizing abdominal pain after 3 months of conservative management with no improvement (Fig. 2).

For the 7 patients with choledocholithiasis, MRCP was done for 5 patients, and ERCP was done for 2 patients. For the 2 patients who have done ERCP, one patient was 9 years old, and ERCP showed mild dilated intrahepatic

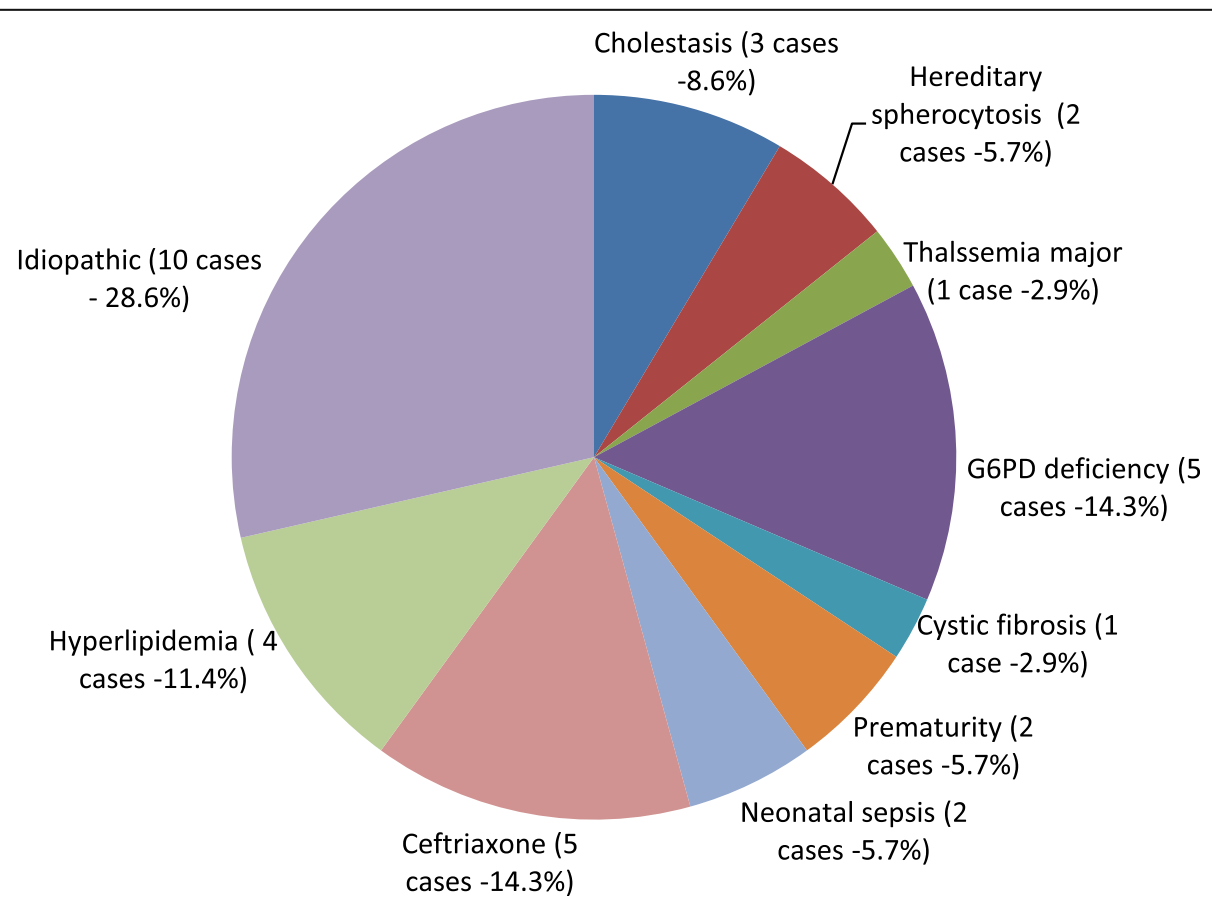

Fig. 1 Possible etiology of gallstones in the study patients 


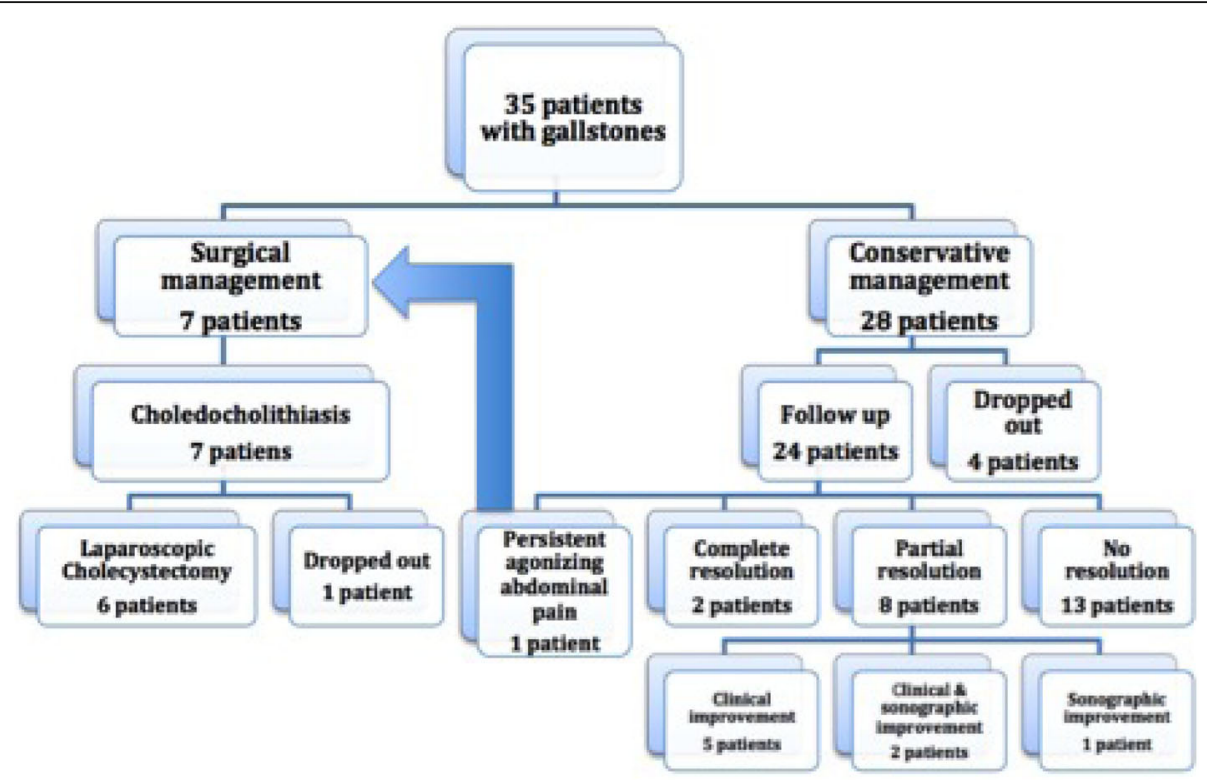

Fig. 2 The management plan of the 35 children with gallstones

biliary radicles (IHBR) and CBD, sphincterotomy with dormia basket was done, and multiple gravels were removed. The other patient was 5 years old; ERCP revealed normal CBD and IHBR with multiple stones, sphincterotomy was done, and stones were removed. Regarding the other 5 patients, one patient was dropped out, while the other 4 patients had spontaneous relief of obstructive jaundice. LC was then performed for the 6 cases. Histopathological analysis of the removed gall bladder in this study was available in 6 cases and revealed chronic cholecystitis in all of them (100\%): none had papillomatosis or adenoma of the gall bladder.

For the 28 patients $(80 \%)$ who were subjected to conservative management, 3 patients dropped out of followup, and one (an infant with cerebral palsy) died 1 month after enrollment due to septicemia. The other 24 patients were followed up for 8 to 34 months with a median of 14 months.

Twelve patients (34.3\%) with symptomatic gallstones were managed conservatively with UDCA for a period ranging from 3 to 24 months and a median of 4.5 months. Follow-up showed complete resolution of gallstones in 2 patients (8.3\%). One of them was a 3-yearold boy with congenital adrenal hyperplasia who showed decrease in number of stones from 2 stones to a single stone after 6 months of therapy; however, this patient showed spontaneous resolution of this remaining gallstone after another 6 months without UDCA administration. Five patients (20.8\%) showed partial improvement, evident with disappearance of symptoms; one patient (4.2\%) showed partial sonographic improvement while 2 patients $(8.3 \%)$ showed partial clinical and sonographic improvement (Fig. 2). Sonographic improvement was based on decreased number of stones and/or disappearance of biliary sludge.

Based on their management strategy, patients were classified into two groups: medical and surgical. Those who needed LC were significantly older at the onset of stones $(P=0.005)$. In addition, their serum cholesterol and LDL levels were significantly higher than those who subjected to medical management (Table 1).

Patients were also divided based on symptomatology at the time of initial diagnosis into two groups: symptomatic and asymptomatic. G6PD deficiency was significantly higher in the asymptomatic group. Symptomatic cases had significant lower RBC count, hemoglobin, and hematocrit than asymptomatic cases (Table 2).

\section{Discussion}

Pediatric cholelithiasis is known to be uncommon. However, recent series documented increased detection rate of gallstones in the pediatric age group [1]. Many risk factors have been described for the development of gallstones in children as hemolytic disorders, prematurity, parenteral nutrition, chronic hepatobiliary diseases, cystic fibrosis, genetic factors, sepsis, ileal diseases, obesity, ceftriaxone use, and abdominal or cardiac surgeries [6]. Idiopathic gallstones, with no identifiable risk factors, have been reported in the literature in the range of $23.2-52.5 \%[2,7,8]$. This is close to the current study where no risk factors were identified in $28.6 \%$ of cases.

Twenty to $40 \%$ of all pediatric gallstone disease can be attributed to hemolytic diseases [9]. Recent studies demonstrated hemolytic diseases in $21.6 \%$ and in $11.8 \%$ of 
Table 1 Risk factors of gallstones and laboratory investigations among medical and surgical patients

\begin{tabular}{|c|c|c|c|}
\hline & Surgical, $n=7(\%)$ & Medical, $n=23(\%)$ & $P$ value \\
\hline Identified risk factors & $5(71.4)$ & $19(82.6)$ & 0.6 \\
\hline \multicolumn{4}{|l|}{ Hemolytic anemia } \\
\hline Hereditary spherocytosis & $1(14.3)$ & $1(4.3)$ & 0.4 \\
\hline Major thalassemia & $0(0)$ & $1(4.3)$ & 1.0 \\
\hline G6PD deficiency & $2(28.6)$ & $7(30.4)$ & 1.0 \\
\hline Dehydration & $1(14.3)$ & $5(21.7)$ & 1.0 \\
\hline Therapy with ceftriaxone & $2(28.6)$ & $4(17.4)$ & 0.6 \\
\hline Family history of gallstones & $1(14.3)$ & $4(17.4)$ & 1.0 \\
\hline Hypertriglyceridemia & $1(14.3)$ & $3(16.7)$ & 1.0 \\
\hline Cholestasis & $0(0)$ & $2(8.7)$ & 1.0 \\
\hline Prematurity & $0(0)$ & $2(8.7)$ & 1.0 \\
\hline Cystic fibrosis & $0(0)$ & $1(4.3)$ & 1.0 \\
\hline Hemoglobin $(g / d l)$ in mean $\pm S D$ & $11.5 \pm 0.95$ & $11.7 \pm 1.2$ & 0.8 \\
\hline T. bilirubin $(N<1.5 \mathrm{mg} / \mathrm{dl})$ median (IQR) & $2.3(1.1-5.1)$ & $0.4(0.2-0.8)$ & $0.002^{*}$ \\
\hline D. bilirubin in median (IQR) & $1.1(0.5-3)$ & $0.1(0.05-0.2)$ & $0.01^{*}$ \\
\hline ALT $(N<40 \mathrm{U} / \mathrm{L})$ in median (IQR) & $92(32-178)$ & $29(20-45)$ & $0.006^{*}$ \\
\hline AST $(N<60 \mathrm{U} / \mathrm{L})$ in median (IQR) & $55(36-125)$ & $37(30-66)$ & 0.1 \\
\hline GGT $(N<30 \mathrm{U} / \mathrm{L})$ in median (IQR) & $105(36-114)$ & $17(11-64)$ & 0.09 \\
\hline AP $(N<640 \mathrm{U} / \mathrm{L})$ in median (IQR) & $252(180-533)$ & $260(192-371)$ & 1.0 \\
\hline INR $(N<1.1)$ in mean $\pm S D$ & $1.04 \pm 0.04$ & $1.1 \pm 0.12$ & 0.4 \\
\hline Cholesterol $(N<200 \mathrm{mg} / \mathrm{dl})$ in mean \pm SD & $180 \pm 47$ & $134 \pm 31$ & $0.006^{*}$ \\
\hline $\mathrm{HDL}(\mathrm{mg} / \mathrm{dl})$ in mean $\pm \mathrm{SD}$ & $38 \pm 12$ & $43 \pm 9.5$ & 0.3 \\
\hline $\mathrm{LDL}(\mathrm{mg} / \mathrm{dl})$ in mean $\pm \mathrm{SD}$ & $120 \pm 44$ & $68 \pm 29$ & $0.002^{*}$ \\
\hline Triglyceride $(N<150 \mathrm{mg} / \mathrm{dl})$ in median (IQR) & $92(62-115)$ & $88(62-119)$ & 0.9 \\
\hline
\end{tabular}

*P value is significant

$A L T$ alanine aminotransferase, $A P$ alkaline phosphatase, $A S T$ aspartate aminotransferase, $D$ direct, GGT gammaglutamyl transpeptidase, G6PD Glucose-6-phosphate dehydrogenase, HDL high-density lipoproteins, INR international randomized ratio, IQR interquartile range, LDL low-density lipoproteins, $R B C s$ red blood cells, $S D$ standard deviation, $T$ total

cases $[10,11]$. In the current study, hemolytic anemia was identified as a risk factor in $34.3 \%$. G6PD deficiency was identified as a risk factor in 9 cases $(25.7 \%)$; this high percentage may be explained by the high prevalence of G6PD deficiency in Egypt as reported by Abo Elella et al. in 2017 who estimated an overall prevalence of G6PD deficiency of 4.3\% among Egyptian neonates [12].

Therapy with ceftriaxone was identified as a risk factor in $17.1 \%$ of cases, in agreement with literature where ceftriaxone use was responsible for $6 \%$ and $27.3 \%$ of cases of gallstones [2, 13].

Chronic biliary disease in the form of cholestasis was present in $8.6 \%$ of our cases, which is close to the results of Dooki and Norouzi (2013) and Della Corte et al (2008) studies $(7.5 \%$ and $5 \%$ respectively) $[2,13]$. It is known that patients with chronic cholestasis especially those with progressive familial intrahepatic cholestasis (PFIC) are prone to cholelithiasis. This is attributed to impaired bile acid secretory function and supersaturation of bile with cholesterol [14]. The three cholestatic patients in this study had normal GGT cholestasis; however, the unavailable genetic analysis in Egypt limited the ability to finally diagnose their specific conditions. These patients might have had PFIC 1 or 2 .

Unlike adults, children are likely to be symptomatic [8]. Our cases showed symptomatic presentation in $62.9 \%$, which is comparable to other studies $[2,9,10]$. On the other hand, the study of Kirsaclioglu et al. [11] showed that symptomatic patients were significantly older than asymptomatic patients, and abdominal pain was the most frequent symptom [11]. Our cases also showed abdominal pain to be the most common symptom in $51.4 \%$.

Complications were documented in $20 \%$ of our cases, which is close to the literature, where they were reported in about $7-20 \%$ of cases [6]. While some older studies reported pancreatitis as the most common complication $[15,16]$, recent studies reported cholecystitis and choledocholithiasis to be more common $[1,11,17]$. Our study showed choledocholithiasis to be more common; meanwhile, none of our 
Table 2 Risk factors of gallstones and complications in symptomatic and asymptomatic patients

\begin{tabular}{|c|c|c|c|}
\hline & Symptomatic, $n=22$ (\%) & Asymptomatic, $n=13(\%)$ & $P$ value \\
\hline Identified risk factors & $14(63.6)$ & $13(100)$ & $0.01^{*}$ \\
\hline \multicolumn{4}{|l|}{ Hemolytic anemia } \\
\hline Chronic hemolytic anemia & $3(13.6)$ & $0(0)$ & 0.274 \\
\hline Glucose-6-phosphate dehydrogenase deficiency & $3(13.6)$ & $6(46.2)$ & 0.050 \\
\hline Dehydration & $6(27.3)$ & $2(15.4)$ & 0.680 \\
\hline Therapy with ceftriaxone & $5(22.7)$ & $1(7.7)$ & 0.377 \\
\hline Family history of gallstones & $2(9.1)$ & $4(30.8)$ & 0.166 \\
\hline Hypertriglyceridemia & $1(4.5)$ & $3(23.1)$ & 0.134 \\
\hline Cholestasis & $1(4.5)$ & $2(15.4)$ & 0.541 \\
\hline Prematurity & $0(0)$ & $2(15.4)$ & 0.131 \\
\hline Cystic fibrosis & $1(4.5)$ & $0(0)$ & 0.435 \\
\hline Choledocholithiasis & $7(31.8)$ & $0(0)$ & $0.015^{*}$ \\
\hline $\mathrm{RBCs}\left(10^{5}\right.$ cells $\left./ \mathrm{mm}^{3}\right)$ in mean $\pm \mathrm{SD}$ & $4.4 \pm 0.5$ & $5 \pm 1$ & $0.047^{*}$ \\
\hline Hemoglobin $(\mathrm{g} / \mathrm{dl})$ in mean $\pm \mathrm{SD}$ & $11.2 \pm 1.4$ & $12.3 \pm 0.8$ & $0.006^{*}$ \\
\hline Hematocrit (\%) in mean $\pm S D$ & $33.8 \pm 3.4$ & $37.5 \pm 3.4$ & $0.008^{*}$ \\
\hline T. bilirubin ( $\mathrm{N}<1.5 \mathrm{mg} / \mathrm{dl})$ median (IQR) & $0.7(0.4-2.6)$ & $0.3(0.2-0.8)$ & 0.021 \\
\hline ALT $(\mathrm{N}<40 \mathrm{U} / \mathrm{L})$ in median (IQR) & $38(27-92)$ & $26(17-40)$ & 0.108 \\
\hline AST $(\mathrm{N}<60 \mathrm{U} / \mathrm{L})$ in median (IQR) & $47.5(36-75)$ & $34(32-71)$ & 0.484 \\
\hline GGT (N < $30 \mathrm{U} / \mathrm{L})$ in median (IQR) & $37.5(12-114)$ & $16(8-28)$ & 0.147 \\
\hline AP (N < $640 \mathrm{U} / \mathrm{L})$ in median (IQR) & $247(190-375)$ & $260(192-371)$ & 0.905 \\
\hline Albumin $(N>3.5 \mathrm{~g} / \mathrm{dl})$ in mean $\pm \mathrm{SD}$ & $4.2 \pm 0.8$ & $3.9 \pm 0.4$ & 0.136 \\
\hline INR $(N<1.1)$ in mean $\pm S D$ & $1.1 \pm 0.1$ & $1.1 \pm 0.1$ & 0.972 \\
\hline
\end{tabular}

*P value is significant

$A L T$ alanine aminotransferase, AP alkaline phosphatase, AST aspartate aminotransferase, GGT gammaglutamyl transpeptidase, INR international randomized ratio, $I Q R$ interquartile range, $R B C$ s red blood cells, $S D$ standard deviation, $T$ total

cases showed pancreatitis or acute cholecystitis. The relatively high complication rate seen in our study group could be explained by the nature of our hospital being a tertiary referral hospital. Severe and complicated cases are commonly referred to our institution, while uncomplicated cases can be managed conservatively in secondary referral centers.

In children, cholecystectomy is recommended for symptomatic and complicated gallstones. It is also recommended for asymptomatic gallstones with chronic hemolytic anemia and asymptomatic large sized gallstones $(>2 \mathrm{~cm})$ for the risk of gallbladder carcinoma [4]. Otherwise, expectant management with periodic clinical and sonographic assessment appears more appropriate [4]. LC was done for 7 of our patients: 6 of them because of choledocholithiasis and one for symptomatic gallstone with agonizing typical right upper quadrant pain. Hajong et al. in 2013 reported 15 non-hemolytic cases of gallstones managed by LC; $80 \%$ were symptomatic, and $13.3 \%$ were asymptomatic while $6.7 \%$ had complicated gallstone in the form of pancreatitis [18]. Another study showed that primary indications for surgery in pediatric population were symptomatic cholelithiasis $(53 \%)$, choledocholithiasis (28\%), and biliary dyskinesia (16\%) [9].
Histopathological analysis of the removed gall bladder (GB) in this study was available in 6 cases and revealed chronic cholecystitis in all of them (100\%); none had papillomatosis or adenoma of GB. This is comparable to the results of Kim et al. study, who showed that, in 24 cases for whom cholecystectomy was performed, histopathology of most cases revealed chronic cholecystitis [19]. On the other side, the removed gallbladders in Della Corte et al. study revealed chronic cholecystitis in $84.6 \%$, papillomatosis in $5.1 \%$, adenoma in $2.6 \%$, and normal GB in $7.7 \%$ [2].

Eighty percent of our cases were managed conservatively, with periodic clinical, laboratory and US followup for 8 to 34 months. None of them developed complications, while 2 cases showed complete spontaneous resolution of gallstones, and 8 cases showed resolution of symptoms and/or sonographic improvement. This may justify the conservative management for uncomplicated cases of gallstones in pediatric population, especially in infants. Jeanty et al., who performed their study on infantile cholelithiasis, observed complete resolution of stones in $25 \%$ of the cases [20], while Gokce et al. [21] reported spontaneous complete resolution in 50\% 
of infantile cases and 19.8\% in older children [1]. This was higher than the resolution rate observed in the current study.

Limitations of this study include the small sample size and the short duration of follow-up in some patients. Moreover, as the study was conducted in a tertiary care unit, many uncomplicated cases may be diagnosed and managed in other centers without referral to our unit, which may explain the higher rate of complications at presentation of our cases.

The strength of this study is that it is the first study to evaluate gallstones among infants and children in Egypt. The absence of a national screening program for G6PD deficiency and other hemolytic anemias, together with high incidence of consanguineous marriages, raises the risk of childhood gallstones in Egypt.

\section{Conclusion}

Risk factors for gallstones could be identified in most of pediatric cases. Symptomatic presentation is common among the pediatric population. Conservative management is supported for asymptomatic cases. Laparoscopic cholecystectomy is a safe and recommended procedure for complicated and symptomatic cases.

\section{Abbreviations}

ALT: Alanine aminotransferase; AST: Aspartate aminotransferase; CBC: Complete blood count; CBD: Common bile duct; PFIC: Progressive familial intrahepatic cholestasis; PT: Prothrombin time; SD: Standard deviation; IHBR: Intrahepatic biliary radicles; INR: International normalized ratio; IQR: Interquartile range; LC: Laparoscopic cholecystectomy; LDL: Lowdensity lipoprotein cholesterol; PT: Prothrombin time; SD: Standard deviation; UDCA: Ursodeoxycholic acid; US: Ultrasonography

\section{Acknowledgements}

Not applicable

\section{Authors' contributions}

EM was responsible for constructing the research hypothesis and planning the methodology. EA was responsible for patients' inclusion and follow-up. ME collected and tabulated the data. AR was responsible for interpretation and presentation of the results. AM and EM wrote the manuscript. ME and EA revised the manuscript. All authors read and approved the final manuscript.

\section{Funding}

This study has no funding source.

\section{Availability of data and materials}

Not applicable

\section{Ethics approval and consent to participate}

This study was performed at Cairo University Hospitals, Cairo, Egypt, after approval of the ethical committee of Kasr Alainy Medical School, Cairo University.Ethical committee number: I-071014.

All procedures followed were in accordance with the ethical standards of the responsible committee on human experimentation (institutional and national) and with the Helsinki Declaration of 1975, as revised in 2008. Written informed consent was obtained from all patients' guardians for being included in the study.

\section{Competing interests}

The authors declare that they have no competing interests.

Received: 29 February 2020 Accepted: 22 May 2020

Published online: 22 July 2020

\section{References}

1. Gupta SK, Shukla VK (2004) Silent gallstones: a therapeutic dilemma. Trop Gastroenterol 25:65-68

2. Della Corte C, Falchetti D, Nebbia G, Calacoci M, Pastore M, Francavilla R et al (2008) Management of cholelithiasis in Italian children: a national multicenter study. World J Gastroenterol 14:1383-1388

3. Garey CL, Laituri CA, Keckler SJ, Ostlie DJ, Stagg HW, Little DC et al (2010) Laparoscopic cholecystectomy in obese and non-obese children. J Surg Res 163:299-302

4. Dipaola F, Heubi J. Disease of gallbladder in infancy, childhood, and adolescence. In: Suchy F, Sokol R, Balistreri W, editors. Liver Disease in Children, 4th edition. Cambridge university press; 2014: 247-264.

5. Alonso M (2004) Gall bladder abnormalities in children with sickle cell disease: management with laparoscopic cholecystectomy. J Pediatr 145: 580-581

6. Svensson J, Makin E (2012) Gallstone disease in children. Semin Pediatr Surg 21:255-265

7. Wesdorp I, Bosman D, de Graaff A, Aronson D, van der Blij F, Taminiau J (2000) Clinical presentations and predisposing factors of cholelithiasis and sludge in children. J Pediatr Gastroenterol Nutr 31:411-417

8. Bogueu C, Murphy A, Gerstle J, Moineddin R, Daneman A (2010) Risk factors, complications, and outcome of gallstones in children: a single center review. J Pediatr Gastroenterol Nutr 50:303-308

9. Mehta S, Lopez M, Chumpitazi B, Mazziotti M, Brandt M, Fishman D (2012) Clinical characteristics and risk factors for symptomatic pediatric gallbladder disease. Pediatrics 129:82-88

10. Sahin Y, Sahin D, Bulut F, Turkut A, Goktepe A (2014) Gallstone in children: aretrospective study of 37 cases in southeast of Turkey. IJSR 3:398-402

11. Kirsaclioglu C, Çakır B, Bayram G, Akbıyık F, Işık P, Tunç B (2016) Risk factors, complications and outcome of cholelithiasis in children: a retrospective, single-centre review. J Paediatr Child Health 52:944-949

12. Abo Elella S, Tawfik M, Barseem N, Moustafa W (2017) Prevalence of glucose-6-phosphate dehydrogenase deficiency in neonates in Egypt. Ann Saudi Med 37:362-365

13. Dooki M, Norouzi A (2013) Cholelithiasis in childhood: a Cohort study in North of Iran. Iran J Pediatr 23:588-592

14. Suchy F, Sundaram S, Shneider B. Familial hepatocellular cholestasis. In: Suchy F, Sokol R, Balistreri W, editors. Liver Disease in Children, 4th edition. Cambridge university press; 2014:199- 215.

15. Reif S, Sloven D, Lebenthal E. Gallstones in children: characterization by age, etiology and outcome. Am J Dis Child 1991;145: 105- 108. PMID: 1898681

16. Lugo-Vicente HL (1997) Trends in management of gallbladder disorders in children. Pediatr Surg Int 12:348-352

17. Sarrami M, Ridley W, Nightingale S, Wright T, Kumar R (2019) Adolescent gallstones-need for early intervention in symptomatic idiopathic gallstones. Pediatr Surg Int 35:569-574

18. Hajong R, Tongper D, Khariong P, Mibang N (2013) Non-hemolytic childhood cholelithiasis managed laparoscopically: a report $1 \mathrm{f} 15$ cases. IOSR-JDMS 7:31-34

19. Kim H, Kim S, Cho Y (2015) Pediatric cholecystectomy: clinical significance of cases unrelated to hematologic disorders. Pediatr Gastroenterol Hepatol Nutr 2:115-120

20. Jeanty C, Derderian S, Courtier J, Hirose S (2015) Clinical management of infantile cholelithiasis. J Pediatr Surg 50:1289-1292

21. Gokce S, Yıldırım M, Erdoğan D (2014) A retrospective review of children with gallstone: Single-center experience from Central Anatolia. Turk J Gastroenterol; 25:46-53.

\section{Publisher's Note}

Springer Nature remains neutral with regard to jurisdictional claims in published maps and institutional affiliations. 